\title{
Multidisciplinary Conferences for Radiology Teaching: Why Medical Students Should See the Significance of Imaging and Lifelong Learning
}

\author{
Ilona Petsch ${ }^{1 *}$, Aglaé Velasco González ${ }^{2}$, and Boris Buerke ${ }^{3}$ \\ ${ }^{1}$ Department of Radiology, Medical Faculty, University of Muenster, and University Hospital \\ Muenster, Muenster, Germany \\ ${ }^{2}$ Department of Radiology, Neuroradiology, Medical Faculty, University of Jena, and University \\ Hospital Jena, Jena, Germany \\ ${ }^{3}$ Head of Department of Diagnostic and Interventional Radiology and MR-Tomography, Klinikum \\ Guetersloh, Guetersloh, Germany, and Department of Radiology, Medical Faculty, University \\ of Muenster, and University Hospital Muenster, Muenster, Germany
}

\section{ARTICLE INFO}

\section{Keywords:}

Multidisciplinary conferences

Radiology

Medical students

Medical teaching

Lifelong learning

\begin{abstract}
Radiology interfaces with all medical disciplines. Whether medical students pursue a career in radiology or any other medical discipline, as future physicians they will always come across imaging at multidisciplinary conferences (MCs). Students at all departments are confronted with imaging in their studies. It is therefore important they recognize the role of radiology in patient care. With imaging being imperative for patient management, radiology is indispensable at MCs. Radiologists cooperate with all clinical departments, and are often final to be asked for decisions at MCs. Joining MCs can prepare students for image interpretation and demonstration in their future clinical practice. MCs can complement to teaching in busy clinical routine. MCs embody educational grounds of lifelong medical learning for radiologists and other physicians. As medical students are going to enter a profession of lifelong learning, MCs can likewise offer valuable educational opportunities. The article presents three reasons to seize MCs as potential teaching opportunities for medical students in radiology: 1. the significance of radiology for MCs; 2. preparation of medical students for MCs; and 3. exploration of MCs for lifelong learning.
\end{abstract}

\section{Introduction}

Teaching medical students continues to be an essential task for radiologists. Why? Radiologists work with physicians from all disciplines as radiology interfaces with all medical disciplines (Gunderman \& Hill, 2012). Buerke et al. stress that patients from practically all clinical departments at hospitals pass through the radiology department (Buerke et al., 2012). Consequently, medical students at all departments are confronted with radiology.

Imaging is imperative for diagnosis (Lim et al., 2017; Oleaga et al., 2019), and necessary for joint clinical decisions on therapy and treatment at multidisciplinary conferences (MCs) (Straus et al., 2014; Rosell et al., 2019). Velasco González et al. emphasize that interventional neuroradiologists do no longer merely provide diagnostic decisions, but are increasingly active in allocating and accompanying therapeutic procedures (Velasco González et al., 2020). Radiologists are often final to be asked before decisions are recorded at MCs.

\footnotetext{
* Corresponding Author E-Mail Address: ipetsch@uni-muenster.de
} 
Radiologists are integral members of MCs (Munk, 2016; O'Keeffe \& Munk, 2018) - with MCs being fundamental for collaborative patient management (Choi \& Filice, 2010; Larson et al., 2017; Alexandersson et al., 2018).

Whether medical students pursue a career in radiology or any other medical discipline, every medical doctor will come across routine imaging (Straus et al., 2014; Darras et al., 2019). Imaging cases demonstrated by radiologists and discussed with physicians at MCs (Collins, 2009; ESR, 2011), equal educational cases for students. Radiologists can facilitate educational opportunities to assist in MC preparation as to prepare students for efficient image interpreting and timely reporting (Oris et al., 2012; Nyhsen et al., 2013; Petsch et al., 2020b), and for a collaborative attitude in MC participation (Lim et al., 2017; Oeppen et al., 2019). Students have to learn that it comes down to extracting complex information for other physicians involved in MCs.

Medical students are going to enter a profession coined by continuous medical development and indispensable lifelong learning (Van Schaik et al., 2013; Burman et al., 2014; Murphy et al., 2014; Steinert \& Macdonald, 2015; Jouhari et al., 2016). MCs embody educational grounds that radiologists and different physicians contribute to in discussions and in often challenging decisions (Mazzaferro \& Majno, 2011; Lesslie \& Parikh, 2017; Saul, 2018). MCs hence offer educational opportunities of lifelong learning for all physicians (Van HoutenSchat et al., 2018; Ramamurthy et al., 2019), and can complement to teaching when high workload hampers additional teaching in already busy clinical routine (ESR, 2011; Pascual et al., 2011; Petsch et al., 2020a).

This article presents three reasons to seize MCs as potential educational opportunities for teaching medical students in radiology elaborating on the: 1. significance of radiology for MCs; 2. preparation of medical students in radiology for MCs; and 3. exploration of MCs for lifelong learning.

\section{Why is radiology significant for MCs?}

Radiological imaging is imperative to provide diagnosis or differential diagnosis (Lim et al., 2017; Oleaga et al., 2019). Imaging is necessary for clinical decisions on the best possible recommendations and therapies as a multidisciplinary consensus at MCs (Rosell et al., 2019), and on follow up of first or further treatments (Choi \& Filice, 2010; O'Keeffe \& Munk, 2018). Radiologists are consulted on the necessity of imaging or radiological interventions, and on other often challenging clinical joint decisions to be made at MCs (Oleaga et al., 2019). Radiologists are therefore integral members of MCs (Croke \& El-Sayed, 2012; Slanetz \& Mullins, 2016) - or play a very active role at conference proceedings. Radiologists contribute to enhanced medical understanding for MC participants (Collins, 2009), aiming at collaborative patient management and improved patient outcome (Mazzaferro \& Majno, 2011; Larson et al., 2017).

Radiology is first in cancer detection (Gunderman et al., 2012), determining tumour invasion and chances of resecting (Lim et al., 2017). Radiology is involved in diagnosis and cancer staging with at least pathology, oncology, radiotherapy, nuclear therapy and surgery (Lesslie \& Parikh, 2017). Radiology is part of long term treatment and patient management (Alexandersson et al., 2018), e.g. if computed tomography (CT) and magnetic resonance imaging (MRI) scans show new findings of pancreatic cystic tumors (Buerke \& Schülke, 2015). Radiology provides other physicians involved in MCs with necessary initial or followup positron emission tomography examinations, e.g. on distant metastases (Buerke et al., 2011; Lim et al., 2017), and explains relevant details on ionizing radiation (Gunderman et al., 2012).

Interventional neuroradiologists perform continuously refining interventions (O'Keeffe \& Munk, 2018), and demonstrate before and after imaging at MCs. They decide on diagnostic and therapeutic procedures in acute neurointerventional events such as strokes (Slanetz \& 
Mullins, 2016), based on routine multimodal brain CT, CT angiography or CT perfusion (Velasco González et al., 2020). Velasco González et al. point out that interventional neuroradiologists no longer merely provide diagnostic decisions where neuroradiological medical treatment would stop, but are increasingly active in allocating and accompanying therapeutic procedures beyond that (Velasco González et al., 2020). Radiologists place venous catheters and feeding tubes (Gunderman \& Hill, 2012), perform image guided minimal invasive punctures, biopsies, and fluid or abscess draining interventions (Gunderman et al., 2012), essential for clinical decisions on further treatment decided on at MCs (Lim et al., 2017).

Gunderman and Hill emphasize the importance of radiology for overall as well as daily patient management (Gunderman \& Hill, 2012). Buerke et al. stress that patients from practically all clinical departments at hospitals pass through the radiology department (Buerke et al., 2012). Radiologists are often final to be asked before decisions are recorded at MCs. So it is not the central question in medical education whether radiology as a discipline receives enough love from medical students. It is rather an important point for future medical doctors to recognize that radiology interfaces with all medical disciplines, and that radiology is therefore indispensable for the field of medicine.

\section{Why prepare students in radiology for MCs?}

Whether medical students pursue a career in radiology or any other medical discipline, every medical doctor will come across radiological imaging (Straus et al., 2014; Alexandersson et al., 2018). Whether work with imaging is routine or occasional as a non-radiologist, basic knowledge in X-ray, CT, MRI and ultrasound interpreting is imperative for medical, dental and veterinary work (Nyhsen et al., 2013; Darras et al., 2019). It is therefore only obvious that radiologists introduce students to routine imaging as future radiologists or non-radiologists (Straus et al., 2014). Giving students the opportunity to have an own idea of imaging preferably in real-life settings such as MCs - also enables them to judge the discipline by their own experience, and come to a more realistic assessment of radiology (Gunderman et al., 2012). If residents in radiology and other departments attend MCs with senior consultant physicians, why should medical students not join. Slanetz et al. suggest residents ought to participate in MCs to prepare for future clinical work (Slanetz \& Mullins, 2016), so there is no profound reason why students should not be invited. With MCs showing potentially valuable teaching opportunities, these can be seized for teaching radiology. At every MC numerous cases read and interpreted by radiologists, are demonstrated by radiologists and discussed in plenary (Collins, 2009; ESR, 2011). For students each demonstration equals an authentic educational case integrated from clinical context - also for physicians (Collins, 2009), and other participants from medical care. Radiologists contribute to an improved and more thorough understanding of radiology, and by this to improved patient management (Gunderman et al., 2012). Particularly students can benefit from such teaching opportunities as to successively learn criteria for normal and abnormal findings in diagnostic imaging, to demonstrate basic clinical patterns in imaging and case reporting (Nyhsen et al., 2013; Petsch et al., 2020a), and to explain indications or contraindications.

Senior consultant radiologists can facilitate educational opportunities by involving students in MC preparation and interaction, and by giving incentives for active contribution (Gunderman $\&$ Bedi, 2013). Assisting tasks in writing up and updating patient lists, or taking notes during MCs, can give a small insight what it means to prepare one to several MCs a day during busy clinical routine. Assisting shows that MC preparation and mandatory participation still needs to take place despite of hampering factors such as workflow (Choi \& Filice, 2010), high workload, staff shortage (ESR, 2011; Petsch et al., 2020a), or covering sick leave. During a global state of emergency such as the ongoing COVID-19 pandemic, MCs around the globe need to continue ensuring patient management and enabling best patient outcome. Assisting 
exemplifies that radiologists interact in numerous MCs maintaining high standard and responsibility (Larson et al., 2017), concurrently attending several clinical duties, plus research and teaching assignments. Assisting shows that radiologists take care of patients, their own residents, and cooperate with colleagues in radiology and other disciplines (Oeppen et al., 2019), and with other medical faculty staff. Assisting offers insight that radiologists do not merely cater medicals students visiting the radiology department but also ones rotating from its "sister disciplines" nuclear medicine and radiotherapy, as well as $\mathrm{PhD}$ students from medical, dental, veterinary or health care studies, and natural or even social sciences.

Radiologists should prepare medical students to develop an efficient work approach in clinical practice (Petsch et al., 2020b), clear communication skills, objectivity and a collaborative attitude during clinical routine essential for every MC participant (Lim et al., 2017; Oeppen et al., 2019). Students in their senior years should be motivated to join MC discussions where appropriate, to learn that imaging is crucial for clinical decisions (Petsch et al., 2020b). It is patient outcome after all that is being discussed and decided on based on radiological images at MCs. Thus, it is important that they are guided in developing techniques to handle image interpreting and to use the appropriate terminology (Rahim \& Ros, 2016). It is crucial that they are trained to report on diagnosis and therapy options effectively (Oleaga et al., 2019). Prompting students in efficient image interpreting and timely reporting should guide them in focusing on relevant information necessary for MC demonstrations (Petsch et al., 2020b). It should become clear that it comes down to extracting complex information necessary for other physicians involved in MCs, and to adjust extracted information to individual patient cases (Gunderman et al., 2012). Radiologists must emphasize that imaging information provided by radiologists is vital for any therapeutic consequence (Gunderman \& Stephens, 2009; Straus et al., 2014).

\section{Why prompt medical students for lifelong learning?}

Medical students are going to enter a profession coined by continuous and rapid development in medical research and practice, hence patient care (Pascual et al., 2011; Steinert \& Macdonald 2015; Oleaga et al., 2019) - a profession as a lifelong learning process (Collins, 2009). Choosing to enter the field of medicine means a lifelong commitment (Gunderman \& Bedi 2013; Murphy et al., 2014), an indispensable prerequisite and responsibility of remaining uptodate in medicine (Van Schaik et al., 2013; Burman et al., 2014). Again, MCs can offer educational grounds for all physicians. One can argue that students ought to enter lifelong learning (Jouhari et al., 2016), or we may say that as future medical doctors they have chosen lifelong development as part of their career and a duty in patient management (O'Keeffe \& Munk, 2018). No matter which discipline will be chosen, learning will always accompany physicians with guidelines to be discussed in evidence-based decision making at MCs according to consistent and timely patient care (Mazzaferro \& Majno, 2011; Burman et al., 2014). MC participants voice individual views or criticism on new implementations, procedures or data presented in medical papers and trial studies (Croke \& El-Sayed, 2012; Lesslie \& Parikh, 2017). Yet they have to agree on aspired procedures as a consensus. Particularly MCs embody educational grounds that radiologists and different physicians cooperatively contribute to and profit from (Collins, 2009; Saul, 2018). Participants receive attendance forms adding to certification relevant for their medical career (Van Schaik et al., 2013; Lesslie \& Parikh, 2017). With additional academic staff other than physicians joining from different medical departments, e.g. academic coordinators, students can see that MCs serve as broader scientific settings generated for educational purposes.

MCs therefore offer wide-ranging opportunities to be explored for educational purposes and lifelong learning early on for medical students (Collins, 2009). MCs can be generated as an addition or implementation in medical education. Yes, medical education already does comprise an enormous amount of knowledge to be acquired (Jouhari et al., 2016). And for 
this reason in particular, MC participation can complement theoretical radiological and medical knowledge in a more thorough and descriptive way. It exemplifies demonstrated cases and clinical procedures, and clarifies tasks of radiologists whom students follow during internships, clerkships and practical training. It explains indications and contraindications for radiological imaging, that might otherwise remain vague for them following radiologists during busy clinical routine in the CT, MRI, ultrasound reading and examinations rooms, in the angiography and interventional neuroradiology sections or in emergency rooms. Because of high workload in private practice, at local hospitals, or university and teaching hospitals, there might be limited time for additional teaching in routine (Van Houten-Schat et al., 2018; Ramamurthy et al., 2019; Petsch et al., 2020a), so that MCs can complement such additional teaching. It shows that image interpreting and clinical decisions made by radiologists during routine must be justified at MCs, and that contributions made to MCs need to be responsible and up-to-date (Mazzaferro \& Majno, 2011; Oleaga et al., 2019). Making students aware of MCs as potential educational opportunities can prepare them for future clinical and medical work of ongoing learning. Inviting them to actively join MCs, adds to a focused learning attitude necessary to prepare for lifelong learning as radiologists and non-radiologists all the same (Oris et al., 2012; Murphy et al., 2014).

\section{Conclusions}

Radiology interfaces with all medical disciplines (Gunderman \& Hill, 2012). Thus, patients from practically all clinical departments pass through radiology for diagnostic, therapeutic and interventional procedures (Buerke et al., 2012; Slanetz \& Mullins, 2016; Oleaga et al., 2019). It is important medical students recognize that radiology is indispensable for recommendations, joint clinical decisions on therapy and treatment, and for collaborative patient management at MCs (Croke \& El-Sayed, 2012; Larson et al., 2017; Alexandersson et al., 2018; Rosell et al., 2019). Radiologists are not merely active and integral members of MCs (Munk, 2016; Lim et al., 2017), but are often final to be asked for decisions. Whether students pursue a career in radiology or any other medical discipline, as future physicians they will always come across radiology at MCs. They should therefore be introduced to routine imaging (Nyhsen et al., 2013; Darras et al., 2019).

MCs can be seized as potential educational opportunities for teaching radiology. Radiologists can facilitate assistance in MC preparation to guide students in effective image interpreting, timely reporting, and in an efficient and collaborative approach in clinical practice (Oris et al., 2012; Rahim \& Ros, 2016; Oeppen et al., 2019). Students have to learn that extracting complex information at MCs is vital for any therapeutic consequence (Straus et al., 2014).

Medical students should join MCs as they are entering a profession of continuous development and ongoing lifelong learning (Collins, 2009; Murphy et al., 2014; Jouhari et al., 2016). They should participate as MCs embody educational grounds of cooperative contribution to patient care (Mazzaferro \& Majno, 2011; Burman et al., 2014; Saul, 2018). If MCs offer lifelong learning opportunities for physicians (Van Houten-Schat et al., 2018; Ramamurthy et al., 2019), why not explore these for students when high workload and staff shortage hamper additional teaching in already busy clinical routine of radiologists (Choi \& Filice, 2010; ESR, 2011; Petsch et al., 2020a).

The three reasons presented to seize MCs as potential teaching opportunities for medical students in radiology, can be supportive in 1. emphasizing the significant role of radiology for MCs; 2. consequently advocating to prepare students for MCs in medical practice; and 3. stressing the benefit of MCs as lifelong learning opportunities.

\section{Disclosure statement}

The authors report no conflicts of interest. The first author and co-authors alone are responsible for the content and writing of this article. 


\section{Acknowledgements}

The authors thank Professor Walter Heindel, director of the Department of Radiology, for contributing to teaching with enthusiasm.

\section{Notes on contributors}

Ilona Petsch, MA Adult Education, Dr. rer. medic candidate, Academic Coordinator, Department of Radiology, University of Muenster, and University Hospital Muenster, Muenster, Germany

Aglaé Velasco González, MD, is a Senior Consultant Neuroradiologist, Interventional Neuroradiologist, Department of Radiology, Neuroradiology, University of Jena, and University Hospital Jena, Jena, Germany

Boris Buerke, MD, MHBA, is Professor and Head of Department of Diagnostic and Interventional Radiology and MR-Tomography, Klinikum Guetersloh, Guetersloh, Germany, and Professor and a Senior Consultant Radiologist, Department of Radiology, University of Muenster, and University Hospital Muenster, Muenster, Germany

\section{List of abbreviations}

1. CT: computed tomography

2. MCs: multidisciplinary conferences

3. MRI: magnetic resonance imaging

\section{References}

Alexandersson, N., Rosell. L., Wihl. J., et al. (2018). Determinants of Variable Resource Use for Multidisciplinary Team Meetings in Cancer Care. Acta Oncol 57(5):675-680. doi.10.1080/0284186X.2017.1400682

Buerke, B. , Gerss, J., Puesken, M., Weckesser, M. (2011). Usefulness of Semi-Automatic Volumetry Compared to Established Linear Measurements in Predicting Lymph Node Metastases in MSCT. Acta Radiol 52(5):540-546. doi.10.1258/ar.2011.100452

Buerke, B., Mellmann, A., Kipp, F., et al. (2012). Hygienic Aspects in Radiology: What the Radiologist Should Know [Hygiene in der Radiologie: Was der Radiologe wissen sollte]. Rofo 184(12):1099-1109. doi.10.1055/s-0032-1325444

Buerke, B., Schülke, C. (2015). Cystic Lesions of the Pancreas [Zystische Tumoren des Pankreas]. Radiologe 55(2):145-156; quiz 157. doi.10.1007/s00117-014-2770-z

Burman, NJ., Boscardin, CK., Van Schaik, SM. (2014). Career-long Learning: Relationship Between Cognitive and Metacognitive Skills. Med Teach 36(8):715-723. doi.10.3109/0142159X.2014.909010

Choi, HH., Filice, RW. (2010). Streamlining Radiologist Workflow for Multidisciplinary Conferences: A Web-Based System to Represent Radiology. $J$ Digit Imaging. doi.10.1007/s10278-019-00317-w

Collins, J. (2009). Lifelong Learning in the 21st Century and Beyond. Radiographics 29(2):613-622. doi.10.1148/rg.292085179

Croke, JM., El-Sayed, S. (2012). Multidisciplinary Management of Cancer Patients: Chasing a Shadow or Real Value? An Overview of the Literature. Curr Oncol 19(4): e232-8. doi.10.3747/co.19.944 
Darras, KE., Spouge, R., Kang, H., et al. (2019). The Challenge with Clinical Radiology Electives: Student and Faculty Perspectives Identify Areas for Improvement. Can Assoc Radiol J 70(4):337-343. doi.10.1016/j.carj.2019.07.004

European Society of Radiology (ESR) (2011). Undergraduate education in radiology. A white paper by the European Society of Radiology. Insights Imaging 2(4):363-374. doi.10.1007/s13244-011-0104-5

Gunderman, RB., Stephens, CD. (2009). Teaching Medical Students About Imaging Techniques. AJR Am J Roentgenol 192(4):859-861. doi.10.2214/AJR.08.1738

Gunderman, RB., Gasparis, PT., Rahman, T. (2012). Educating Medical Students About Radiologists' Contributions to Patient Care. Acad Radiol 19(7):908-9909. doi.10.1016/j.acra.2012.03.016

Gunderman, RB., Hill, DV. (2012). Student Concerns and Misconceptions about A Career in Radiology. Acad Radiol 19:366-368. doi.10.1016/j.acra.2011.10.028

Gunderman, RB., Bedi, HS. (2013). The Two-Minute Teacher. Acad Radiol 20(12):16101612. doi.10.1016/j.acra.2013.04.021

Jouhari, Z., Haghani, F., Changiz, T. (2016). Assessment of Medical Students' Learning and Study Strategies in Self-Regulated Learning. Adv Med Educ Prof 4(2):72-79

Larson, DB., Donnelly, LF., Podberesky, DJ., et al. (2017). Peer Feedback, Learning, and Improvement: Answering the Call of the Institute of Medicine Report on Diagnostic Error. Radiology 283(1):231-241. doi.10.1148/radiol.2016161254

Lesslie, M., Parikh, JR. (2017). Implementing a Multidisciplinary Tumor Board in the Community Practice Setting. Diagnostics 7(4):55. doi.10.3390/diagnostics7040055

Lim, RS., Kielar, AZ., El-Maraghi, RH., et al. (2017). Multidisciplinary Retroperitoneal and Pelvic Soft-Tissue Sarcoma Case Conferences: The Added Value That Radiologists Can Provide. Curr Oncol 24(3):e171-e175. doi.10.3747/co.24.3478

Mazzaferro, V., Majno, P. (2011). Principles for the Best Multidisciplinary Meetings. Lancet Oncol 12(4):323-325. doi.10.1016/S1470-2045(11)70010-9

Munk, PL. (2016). A Place in the Sun: The Importance of Radiology in Multidisciplinary Conference. Can Assoc Radiol J 67(3):203. doi.10.1016/j.carj.2016.06.002

Murphy, KP., Crush, L., O’Malley, E., et al. (2014). Medical student knowledge regarding radiology before and after a radiological anatomy module: implications for vertical integration and self-directed learning. Insights Imaging 5(5):629-34. doi.10.1007/s13244-0140346-0

Nyhsen, CM., Steinberg, LJ., O’Connell, JE. (2013). Undergraduate radiology teaching from the student's perspective. Insights Imaging 4(1):103-109. doi.10.1007/s13244-012-0206-8

Oeppen, RS., Davidson, M., Scrimgeour, DS., et al. (2019). Human Factors Awareness and Recognition During Multidisciplinary Team Meetings. J Oral Pathol Med 48(8):656-661. doi.10.1111/jop.12853 
O'Keeffe, M., Munk, PL. (2018). Radiologists: A Keystone of Multidisciplinary Oncology Practice. Can Assoc Radiol J 69(1):1. doi.10.1016/j.carj.2018.01.001

Oleaga, L., Dewey, M., Iezzi, R., et al. (2019). ESR statement on new approaches to undergraduate teaching in Radiology. Insights Imaging 10(1):109. doi.10.1186/s13244-019-08049

Oris, E., Verstraete, K., Valcke, M. (2012). Results of a survey by the European Society of Radiology (ESR): undergraduate radiology education in Europe-influences of a modern teaching approach. Insights Imaging 3(2):121-30. doi.10.1007/s13244-012-0149-0

Pascual, TNB., Chhem, R., Wang, SH., Vujnovic, S. (2011). Undergraduate Radiology Education in the Era of Dynamism in Medical Curriculum: An Educational Perspective. Eur $J$ Radiol 78(3):319-325. doi.10.1016/j.ejrad.2010.08.039

Petsch, I., Velasco González, A., Heindel, W., Buerke, B. (2020a). Making Medical Teaching Interactive: Catering Learning Needs in Radiology. In: Abstracts of ARETL 2020 Virtual Conference; Berlin, Germany, 2020 March 20-22. Abstract 370. 2nd International Conference on Advanced Research in Education, Teaching and Learning: Vilnius, Lithuania. https://www.dpublication.com/abstract-of-2nd-aretl/62-ar/

Petsch, I., Velasco González, A., Heindel, W., Buerke, B. (2020b). Teaching radiology: Integrating medical students by increasing communication. In: Abstracts of ARETL 2020 Virtual Conference; Berlin, Germany, 2020 March 20-22. Abstract 372. 2nd International Conference on Advanced Research in Education, Teaching and Learning: Vilnius, Lithuania. https://www.dpublication.com/abstract-of-2nd-aret1/62-ar/

Rahim, S., Ros, P. (2016). Moving Away from Spoon-Feeding as a Teaching Style in Radiology. AJR Am J Roentgenol 207(6):1232-1238. doi.10.2214/AJR.16.16694

Ramamurthy, S., Er, HM., Nadarajah, VD., Radhakrishnan, AK. (2019). Medical students orientation toward lifelong learning. Med Teach 13;1-6. doi. 10.1080/0142159X.2019.1646894

Rosell, L., Wihl, J., Hagberg, O., et al. (2019). Function, Information, and Contributions: An Evaluation of National Multidisciplinary Team Meetings for Rare Cancers. Rare Tumors 11:2036361319841696. doi.10.1177/2036361319841696

Saul, D. (2018). What It Means 'To Teach' as a Radiologist in the Modern Era: A Personal Perspective. Pediatr Radiol 48(10):1381-1387. doi.10.1007/s00247-018-4201-1

Slanetz, PJ., Mullins, ME. (2016). Radiology Education in the Era of Population-based Medicine in the United States. Acad Radiol 23(7):894-897. doi.10.1016/j.acra.2016.01.017

Steinert, Y., Macdonald, ME. (2015). Why Physicians Teach: Giving Back by Paying It Forward. Med Educ 49(8):773-782. doi.10.1111/medu.12782

Straus, CM., Webb, EM., Kondo, KL., et al. (2014). Medical Student Radiology Education: Summary and Recommendations from a National Survey of Medical School and Radiology Department Leadership. J Am Coll Radiol 11(6):606-610. doi.10.1016/j.jacr.2014.01.012

Van Houten-Schat, MA., Berkhout, JJ., Van Dijk, N., Endedijk, MD., et al. (2018). Selfregulated Learning in the Clinical Context: A Systematic Review. Med Educ 52(10):10081015. doi.10.1111/medu.13615 
Van Schaik, SM., Plant, J., O'Sullivan, P. (2013). Promoting Self-Directed Learning Through Portfolios in Undergraduate Medical Education: The Mentors' Perspective. Med Teach 35(2):139-144. doi.10.3109/0142159X.2012.733832

Velasco González, A., Buerke, B., Görlich, D., et al. (2020). Clot Analog Attenuation in Noncontrast CT Predicts Histology: An Experimental Study Using Machine Learning. Transl Stroke Res. doi.10.1007/s12975-019-00766-z 